\title{
Psicoterapia de grupo com foco na adaptação acadêmica: um experimento com estudantes
}

\section{Group psychotherapy with focus on academic adaptation: an experimont with students}

\section{Psicoterapia de grupo con foco en la adaptación académica: un experimento con estudiantes}

\author{
Daiane Bocard do Couto* \\ Universidade Federal Rural do Rio de Janeiro - UFRRJ, Seropédica, Rio de Janeiro, \\ Brasil
}

\section{Carla Cristine Vicente**}

Universidade Federal Rural do Rio de Janeiro - UFRRJ, Seropédica, Rio de Janeiro, Brasil

\begin{abstract}
RESUMO
O estudo teve foco na adaptação acadêmica para identificar efeitos da psicoterapia de grupo na elaboração do processo de adaptação em estudantes no primeiro ano da universidade. Participaram do estudo 24 estudantes, quatro do sexo masculino e 20 do sexo feminino, com idades entre 18 e 29 anos, do curso de psicologia da Universidade Federal Rural do Rio de Janeiro. Os participantes foram divididos em dois grupos de 12 pessoas, chamados de grupo A e grupo B. O grupo A frequentou 8 sessões de psicoterapia de grupo. O grupo B funcionou como grupo controle. Ambos os grupos responderam o Questionário de Vivências Acadêmicas reduzido QVAr, em dois momentos, no início e no final do período letivo. Os resultados mostraram que o grupo que participou da psicoterapia, ao final do estudo, elevou seu nível de adaptação acadêmica, passando de médio adaptado para bastante adaptado. Enquanto o grupo controle regrediu seu nível de adaptação, indo de bastante adaptado para médio adaptado. Concluiu-se que participação engajada em um grupo psicoterápico auxiliou estudantes a enfrentar seu processo de adaptação acadêmica no 10 ano de graduação.
\end{abstract}

Palavras-chave: adaptação acadêmica, psicoterapia de grupo, QVA-R.

\section{ABSTRACT}

The study focused on academic adaptation to identify effects of group psychotherapy in the adaptation process of students in the first year of university. The sample consisted of 24 students, 04 males and 20 females, aged 18 and 29 years old, from the psychology course at the Universidade Federal Rural do Rio de Janeiro. Participants were divided into two groups of 12 people each, called group A and group B. Group A attended 8 group psychotherapy sessions. Group B served as control group. Both groups answered the Academic Experiences Questionnaire reduced - QVAr, on two 
occasions, one at the beginning and another at end of the school year. The results showed that the group who participated in Psychotherapy, at the end of the study, raised its level of academic adaptation, from medium adapted to quite adapted. While the control group declined on their level of adaptation, going from quite adapted to medium adapted. The study concluded that engaged participation in a psychotherapeutic group helped students to face their academic adaptation process in the 1st year of graduation.

Keywords: academic adaptation, group psychotherapy, QVA-R.

\section{RESUMEN}

El estudio se centró en la adaptación académica para identificar efectos de la psicoterapia de grupo en la elaboración del proceso de adaptación de estudiantes del primer año de universidad. La muestra estuvo conformada por 24 estudiantes, 4 hombres y 20 mujeres, de entre 18 y 29 años, de la carrera de psicología de la Universidad Federal Rural de Río de Janeiro. Los participantes fueron divididos en dos grupos de 12 personas, llamados grupo A y B. Los participantes del grupo A asistieron a 8 sesiones de psicoterapia de grupo. El Grupo B sirvió como grupo de control. Ambos grupos respondieron el Cuestionario de Vivencias Académicas reducido- QVA-r, en dos ocasiones, al principio y al final del año escolar. Los resultados mostraron que el grupo que participó de la psicoterapia al final del estudio, elevó su nivel de adaptación académica, pasando de la categoría de medio adaptado a bastante adaptado. Por su parte, el grupo de control retrocedió en su nivel de adaptación, yendo de la categoría bastante adaptado a medio adaptado. Se concluyó que la participación comprometida en un grupo psicoterapéutico contribuyó a que los estudiantes cumplan con su proceso de adaptación académica en el primero año de la carrera.

Palabras clave: adaptación académica, psicoterapia de grupo, QVA-R.

\section{I ntrodução}

Ao ingressarem no ensino superior, os estudantes se deparam com um ambiente social diferente dos que estavam habituados, exatamente no momento em que passam pela complicada tarefa de tornar-se adulto. As novas regras, pessoas e situações exigem adaptação do ingressante ao longo do primeiro ano na universidade, a fim de que possa se fixar e permanecer.

Ao analisar o processo de adaptação acadêmica, é perceptível a existência de inúmeros fatores que podem dificultar o enfrentamento das dificuldades características do início de curso de graduação por jovens estudantes. Várias situações podem gerar crises, comprometendo o desenvolvimento e o rendimento acadêmico, a saber: a imersão em um novo sistema didático e em um novo círculo social; a possível saída da casa dos pais; aumento de responsabilidade sobre sua própria vida e decisões pessoais; a ampliação da liberdade e ter que administrar a própria vida financeira (Pascarella \& Terenzini, 2005; Diniz \& Almeida, 2006; Teixeira, Dias, Wottrich \& Oliveira, 2008). 
Para que o indivíduo possa desenvolver suas potencialidades de forma plena, é necessário que esteja adaptado ao meio ao qual está inserido. O processo de adaptação é percebido de forma particular por quem o enfrenta. Assim, para o indivíduo que apresente dificuldade de lidar com os fatores envolvidos no próprio processo de adaptação acadêmica, ou seja, não se adapte à universidade, consequências prejudiciais podem se manifestar, como a reprovação em disciplinas ou o abandono do curso de graduação, o que para a instituição de ensino gera o aumento do número de discentes que não concluem seus cursos ou evadem da instituição (Ferreira, Almeida \& Soares, 2001; Bardagi \& Hutz, 2005; Cunha \& Carrilho, 2005).

Nesse estudo, a participação em grupo de psicoterapia foi utilizada como estratégia de organização frente às dificuldades e possibilidade de êxito na adaptação acadêmica pela sua reconhecida eficácia no auxílio a mudanças de perceptivas e de comportamento (Souza, 2006). A psicoterapia de grupo apresenta efeitos favoráveis em períodos relativamente curtos, variando de poucos meses a dois anos, segundo Bechelli e Santos (2002). De modo geral, o estudo de Howard, Kopta, Krause e Orlinsky (1986) demonstrou que as investigações sobre intervenção psicoterápica têm evidenciado que após sete sessões, pelo menos $50 \%$ dos clientes apresentam melhora, após catorze sessões $75 \%$, e para que haja mudanças de comportamentos em casos mais graves, vinte e uma sessões. Enéas, Faleiros e Sá (2000), descreveram que não há consenso a respeito do número de sessões, e encontraram indicações variando de quatro até 40 sessões, sendo 12 sessões o número sugerido com mais frequência.

Ao longo da vida, indivíduos estabelecem variados vínculos grupais, o que favorece o estabelecimento do grupo como recurso terapêtico que pode auxiliar o estudante a fazer contato com conteúdos internos, comportamentos, papéis sociais desempenhados por ele, expectativas em relação à universidade e percepções diante das dificuldades na convivência com outros colegas e professores.

Em grupos terapêuticos, os participantes apoiam-se uns aos outros, compartilham angústias e encontram pessoas com conflitos semelhantes, o que contribui para o processo de crescimento pessoal. Dessa forma, o grupo funciona como um espaço de trocas, capaz de espelhar para cada participante aquilo que este ainda não conseguiu perceber a seu próprio respeito, auxiliando-o em sua autocompreensão. Na psicoterapia de grupo, cada participante pode ser também considerado um agente terapêutico para os demais. Esta pode proporcionar ao cliente autoconhecimento, suporte para lidar com as demandas internas, alívio de tensões, mudanças de percepção e de comportamento, manutenção da saúde mental, crescimento pessoal, melhora nas relações interpessoais, ampliação 
das possibilidades de reorganização da própria vida, entre os principais benefícios (Yalom \& Leszcz, 2007). Nesse sentido, o emprego da psicoterapia de grupo objetivou oferecer aos alunos suporte psicológico, favorecido pelo contato com pares que enfrentam situações semelhantes no processo de adaptação acadêmica.

\section{Adaptação Acadêmica}

Em psicologia, o termo adaptação se refere a uma relação entre o indivíduo e seu meio social (Moraes, 2003). À medida que o ser humano se desenvolve, ingressa em novos grupos sociais. Esse movimento de inserção pode exigir que o indivíduo passe por um processo de adaptação. Especificamente, a adaptação acadêmica é um processo complexo e multidimensional, que envolve diferentes fatores de difícil articulação, tanto de natureza intrapessoal, como de natureza contextual. Engloba as vivências do estudante ao longo de sua formação e a interação contínua e dinâmica entre suas características pessoais e o ambiente da universidade (Fernandes \& Almeida, 2005; Soares, Almeida, Diniz \& Guisande, 2006; Carmo \& Polydoro, 2010; Sarriera, Paradiso, Schütz \& Howes, 2012).

De acordo com o Instituto Nacional de Estudos e Pesquisas Educacionais Anísio Teixeira - INEP, o último Senso da Educação Superior de 2013 apontou que o total de alunos que ingressou no ensino superior foi de 2,7 milhões. Considerando-se o período 20032013, o número de ingressantes em cursos de graduação aumentou $76,4 \%$. Entretanto, apesar de os dados sobre o ingresso mostrar que este mais que dobrou no período citado, quando se considera o número de diplomados nos cursos de graduação presencial, o que se observa é que o número de alunos que tem ingressado nas universidades do país é desproporcional ao número dos que se formam. Um dos fatores que podem contribuir para esse quadro é a elaboração pessoal inadequada do processo de adaptação acadêmica (Ministério da Educação, INEP, 2013).

Vale dizer que o ingresso na universidade não garante o êxito educacional do estudante. As características e exigências do nível superior de ensino exigem uma mudança de comportamento. A vivência de ser estudante pode causar insegurança quanto à carreira e exige mudança significativa de hábitos. Além disso, pode haver decepções quanto às expectativas levantadas em relação à vida universitária, à estrutura e metodologia do trabalho acadêmico.

Alguns estudos, como os de Palma, Palma e Brancaleoni (2005) e de Bardagi (2007), têm revelado altos índices de insatisfação com a escolha do curso de graduação entre os acadêmicos. Um dos resultados encontrados por Bardagi e Hutz (2012) foi relativo a relatos de decepção por parte dos alunos em relação a expectativas 
do que imaginavam encontrar quando ingressaram na graduação. Essa decepção ocorreu no âmbito das relações interpessoais. Por sua vez, Oliveira e Morais (2015) apontaram em seu trabalho que os estudantes têm tido dificuldades de adaptação relacionadas a duas áreas: a pessoal e o estudo.

Há uma mudança significativa no ambiente educacional do ensino médio em relação ao ensino superior. O modo como os alunos se integram ao contexto do ensino superior, segundo Teixeira et al. (2008), faz com que eles tenham a possibilidade de aproveitar as oportunidades oferecidas pela universidade para sua formação profissional e seu desenvolvimento psicossocial.

De acordo com Soares e Prette (2015), a universidade é um ambiente complexo que requer a integração e harmonização de processos cognitivos, sociais e afetivos, com demandas para pensar, relacionar-se com os outros e gostar do que se faz. O ingresso na universidade pode então, segundo Teixeira et al. (2008), representar uma tarefa de desenvolvimento típica da transição para a vida adulta. Nota-se que a experiência universitária não se restringe à formação profissional, sendo também permeada pelas experiências existenciais, que afetam o jovem no enfrentamento das transformações e questões relacionadas ao seu crescimento pessoal, autoconhecimento, integração social e identificação grupal (Diniz \& Almeida, 2006; Feitosa, 2001, Soares et al., 2006). Marinho-Araujo, Fleith, Almeida, Bisinoto e Rabelo (2015) encontraram que os estudantes ingressantes nos cursos de graduação têm expectativas por uma formação de qualidade que os leve ao sucesso profissional, bem como ao ingresso no ensino superior que favoreça e potencialize seu desenvolvimento pessoal.

O círculo de amizade muda, havendo a necessidade de estabelecer novos vínculos. Enquanto tais vínculos não se estabelecem, o jovem conta apenas com seus próprios recursos psicológicos e o apoio das redes formadas anteriormente ao ingresso na universidade, como amigos antigos e familiares, para enfrentar eventuais dificuldades que possam surgir (Teixeira et. al., 2008). Nessa perspectiva, ajustar-se à universidade implica integrar-se socialmente com as pessoas desse novo contexto, participando de atividades sociais e desenvolvendo relações interpessoais satisfatórias (Diniz \& Almeida, 2006; Pascarella $\&$ Terenzini, 2005; Teixeira et. al., 2008).

Cumpre salientar que o relacionamento interpessoal é um tópico recorrente nos estudos que se dedicam a investigar o processo de adaptação universitária. Oliveira, Dantas, Azevedo e Banzato (2008) realizaram um levantamento do serviço de saúde mental da Unicamp e identificaram que a maior parte das queixas dos 2.194 alunos que procuraram o serviço, estavam relacionadas a problemas de relacionamento interpessoal. Bardagi e Hutz, (2012) salientam a importância dos relacionamentos interpessoais na graduação, com 
colegas e com professores, em especial no período inicial do curso universitário.

\section{Psicoterapia de Grupo}

A psicoterapia de grupo é um processo destinado a qualquer pessoa que pretenda expandir sua autoconsciência e não apenas às que apresentam alguma psicopatologia. $O$ participante traduz seus insights em informação que faça sentido para sua vida.

Segundo Bechelli e Santos (2002), estudos indicam que a psicoterapia de grupo se equipara com a psicoterapia individual em critério de eficácia e não são excludentes entre si. Já Bock (2001), considera que a modalidade de atendimento grupal é privilegiada em relação ao trabalho individual devido à diversidade e heterogeneidade presente e por acreditar que a dinâmica estabelecida enriquece o processo terapêutico facilitando a observação dos valores, dificuldades, opiniões, interesses e projetos de vida do outro.

Zimerman (2000) aponta que um grupo pode ser classificado de acordo com a sua vertente teórica, como o tipo de setting instituído, com a finalidade a ser alcançada, com o tipo dos integrantes e com o tipo de vínculo com o coordenador.

Não existe um padrão fixo e único de características da psicoterapia de grupo. Os grupos psicoterapêuticos podem trabalhar com as demandas que surgirem por parte dos clientes ou podem ter temáticas específicas. Os grupos podem variar de tamanho, de oito a 20 membros, visando à estimulação da interação (Yalom \& Leszcz, 2007). E a respeito do tempo de duração das sessões em grupo, Meier e Kudlowiez (2003), propõem sessões de uma a duas horas, para que os integrantes não se desgastem.

Os grupos terapêuticos tendem a proporcionar que os participantes, na condição de co-terapeutas, possam se perceber em situações próximas ou distintas de outros participantes, interagindo a partir de seus sentimentos e percepções, o que amplia sua consciência de si e também a autoestima associada ao sentimento de pertencimento (Bechelli \& Santos, 2002).

Diante dos apontamentos supracitados, este estudo tem o seguinte questionamento norteador: a participação em um grupo psicoterápico pode auxiliar o estudante a enfrentar e elaborar o próprio processo de adaptação acadêmica no primeiro ano de graduação? 


\section{Método}

Trata-se de um estudo experimental, com delineamento correlacional, sendo composto por duas fases, cujos objetivos, reiteramos, foram identificar os efeitos da participação em psicoterapia de grupo na adaptação acadêmica.

Participaram do estudo vinte e quatro estudantes, quatro do sexo masculino e vinte do sexo feminino, com idades entre dezoito e vinte e nove anos, do primeiro ano do curso de psicologia, de horário integral da Universidade Federal Rural do Rio de Janeiro (UFRRJ), residentes nos municípios do Rio de Janeiro e Seropédica, escolhidos por sorteio dentre um grupo de voluntários. Os participantes foram divididos em dois grupos de doze pessoas, chamados de grupo A e grupo B. O grupo A foi composto por alunos que frequentaram semanalmente, durante oito semanas, um grupo de psicoterapia formado especificamente para este fim, nas dependências da universidade. Os encontros tiveram duração de duas horas e foram facilitados pelas autoras. Estes privilegiaram temáticas relativas à adaptação acadêmica, como relacionamentos interpessoais, organização do tempo, autonomia, habilidades de carreira, conhecimentos institucionais e percepção de mudanças. Assim como, os conteúdos que emergiram espontaneamente no grupo. O grupo B funcionou como grupo controle e os alunos não participaram das sessões de psicoterapia de grupo. Ambos os grupos responderam o Questionário de Vivências Acadêmicas reduzido - QVAr, em dois momentos, o primeiro, no início do período letivo e o segundo, ao final do período letivo, ou seja, antes e depois de o grupo A participar da psicoterapia de grupo. As sessões de psicoterapia de grupo aconteceram em sala adequada para este fim.

O referencial teórico que fundamentou as sessões psicoterápicas do grupo foi a orientação Fenomenológica Existencial Humanista. A proposta foi facilitar o "experenciar" do próprio modo de organização, um olhar para si mesmo, como forma de fabricar o sentido de ser (Erthal, 2013). Essa abordagem compreende o indivíduo como sujeito ativo na elaboração do seu projeto de vida, sendo função do terapeuta acompanha-lo em seu processo de reflexão, facilitando que este elabore sua identidade e faça escolhas de maneira consciente, madura, autônoma e presente. A teia de relações no grupo reproduz as interações na vida, o que permite organizar a experiência de ser adulto, estudante universitário, responsável e gestor de sua própria história. Alguns pilares trabalhados nessa abordagem são: escuta ativa, empatia, aceitação, sentido da vida, autenticidade, projeto de vida, liberdade, responsabilização (Sartre, 2005).

Durante as sessões foram realizadas técnicas de dinâmica de grupo, com o objetivo de fazer emergir sentimentos que pudessem ser elaborados pelos participantes do grupo. As técnicas utilizadas foram 
retiradas e adaptadas da literatura existente (Militão \& Militão, 2000; Serrão \& Baleeiro, 1999).

O instrumento utilizado nesse estudo foi o Questionário de Vivências Acadêmicas reduzido - QVAr (elaborado por Almeida, Ferreira \& Soares, em 1999 e adaptado para amostras brasileiras por Santos, Noronha, Amaro, \& Villar, 2005). Através deste, buscou-se avaliar a autopercepção dos alunos sobre o próprio processo de adaptação antes e depois de participarem das sessões de psicoterapia de grupo. Para a aplicação desse instrumento foi feita uma comunicação pessoal com cada participante instruindo sobre o preenchimento do questionário via internet nos dois momentos.

A análise do QVAr foi realizada por meio do teste $t$ de Student $(p<0,05)$, a fim de comparar os resultados entre os 1 을 2 은 momentos e entre os grupos A e B. A análise também envolveu uma estatística descritiva dos resultados por dimensões e itens do QVA-r. Diversos estudos que abordam esse tema evidenciam o QVAr como adequado para a verificação da adaptação universitária (Villar, 2001; Almeida, Ferreira \& Soares, 1999; Granado, Santos, Almeida, Soares $\&$ Guisande, 2005; Casanova \& Polydoro, 2010). Este instrumento é uma escala de autorrelato que tem por intuito avaliar a forma como os jovens vivenciam algumas das exigências da vida acadêmica (Granado et. al., 2005; Casanova \& Polydoro, 2010). Sua escala é do tipo likert de resposta, variando de 1 a 5 pontos, na qual o (1) corresponde à afirmação nada a ver comigo; (2) pouco a ver comigo; (3) algumas vezes de acordo comigo; (4) bastante a ver comigo e (5) tudo a ver comigo.

O QVAr foi desenvolvido em Portugal, partindo de uma versão expandida (QVA), que continha cento e setenta itens. Esse estudo utilizou a versão reduzida (QVAr), adaptada ao contexto brasileiro e validada para a população brasileira, composta por cinquenta e cinco itens distribuídos em cinco dimensões: Pessoal, Interpessoal, Carreira, Estudo e Institucional. A dimensão pessoal refere-se ao bem-estar físico e psicológico; aborda aspectos como o equilíbrio emocional, a estabilidade afetiva, o otimismo, a tomada de decisões e a autoconfiança. A dimensão interpessoal refere-se às relações com os colegas e a competências de relacionamento em situações de maior intimidade; inclui o estabelecimento de amizades, a procura de ajuda. A dimensão carreira refere-se a sentimentos relacionados com o curso frequentado e perspectivas de carreira; inclui a satisfação com o curso e percepção de competências para o curso. A dimensão estudo refere-se a hábitos de estudo e à gestão de tempo; inclui as rotinas de estudo, o planejamento do tempo, a utilização de recursos de aprendizagem, a preparação para as provas. A dimensão institucional refere-se à apreciação da instituição de ensino frequentada; inclui os sentimentos relacionados à instituição, o desejo de permanecer ou mudar de instituição, conhecimento e 
apreciação das infraestruturas (Almeida, Soares \& Ferreira, 2002; Igue, Bariani \& Milanesi, 2008; Casanova et. al., 2010).

É válido ressaltar que o QVAr contém dezessete itens cujas afirmações têm sentido negativo e que devem ser invertidos na planilha, no momento da análise. Neste estudo, foi feita menção a esses itens como "itens invertidos".

Sobre os procedimentos éticos deste estudo, os participantes assinaram o Termo de Consentimento Livre e Esclarecido, contendo as informações sobre a pesquisa. Foram informados sobre os possíveis riscos e benefícios decorrentes da participação na pesquisa, sobre sigilo das identidades e sobre a possibilidade da desistência a qualquer momento, sem que isto pudesse lhes gerar qualquer prejuízo pessoal, institucional ou financeiro, bem como também a ausência de compensação financeira pela participação. Eles também receberam a informação que os dados seriam utilizados em divulgações científicas. O projeto deste estudo foi submetido ao comitê de ética da UFRRJ e sua aprovação gerou o processo no. 2308.010211/2014-97.

\section{Resultados e Discussão}

São apresentadas as médias das cinco dimensões avaliadas no questionário QVAr, nos grupos A e B e nos dois diferentes momentos em que se aplicou o questionário (tabela 1 ).

A dimensão Pessoal não apresentou diferença significativa quando comparados os grupos A (experimental) e B (controle) no momento 2 (final do experimento). Provavelmente esse resultado ocorreu devido ao grupo $B$ ter apresentado média maior do que o grupo $A$, no momento 1 (início do experimento). O grupo $B$ também não diferiu estatisticamente quando comparados os momentos 1 e 2 . No entanto, nota-se que o grupo $\mathrm{A}$ aumentou sua média discretamente, quando comparados os momentos 1 e 2 (tabela 1), indicando, assim, que participar da psicoterapia de grupo pode ter influenciado na percepção da dimensão Pessoal dos participantes, mesmo que o resultado não tenha sido significativo. Teixeira et. al. (2008) afirmam que a entrada na universidade é uma experiência que impacta o estudante, não apenas pelas demandas do ambiente universitário, mas também porque provoca uma mudança mais radical no contexto de vida pessoal do jovem, exigindo o desenvolvimento de respostas adaptativas frente a um conjunto de situações desafiadoras relacionadas ao gerenciamento da própria vida. Estudos que avaliaram a prestação de serviços de apoio psicológico no contexto universitário relataram os efeitos positivos das intervenções em promover o desenvolvimento pessoal dos estudantes (Dias, 2006; Pereira et al., 2006; Ferreira, 2009). 
Tabela 1: Médias dos índices de escala de cada dimensão avaliada antes e após a terapia de grupo

\begin{tabular}{|c|c|c|c|c|c|c|}
\hline & \multicolumn{2}{|c|}{ Pessoal } & \multicolumn{2}{|c|}{ Interpessoal } & \multicolumn{2}{|c|}{ Carreira } \\
\hline & Momento 1 & Momento 2 & Momento 1 & Momento 2 & Momento 1 & Momento 2 \\
\hline Grupo A & $40,3 \mathrm{Aa}$ & $45,5 \mathrm{Aa}$ & $39,4 \mathrm{Ba}$ & $44,3 \mathrm{Aa}$ & $43,0 \mathrm{Aa}$ & $43,9 \mathrm{Aa}$ \\
\hline \multirow[t]{3}{*}{ Grupo B } & $45,8 \mathrm{Aa}$ & $45,3 \mathrm{Aa}$ & $42,5 \mathrm{Aa}$ & $42,6 \mathrm{Aa}$ & $44,3 \mathrm{Aa}$ & $42,6 \mathrm{Aa}$ \\
\hline & \multicolumn{2}{|c|}{ Estudo } & \multicolumn{2}{|c|}{ Institucional } & \multicolumn{2}{|c|}{ Geral } \\
\hline & Momento 1 & Momento 2 & Momento 1 & Momento 2 & Momento 1 & Momento 2 \\
\hline Grupo A & $26,3 \mathrm{Aa}$ & $26,8 \mathrm{Aa}$ & $25,1 \mathrm{Aa}$ & $26,2 \mathrm{Aa}$ & $177,3 \mathrm{Ba}$ & $190,6 \mathrm{Aa}$ \\
\hline Grupo B & $30,3 \mathrm{Aa}$ & $28,3 \mathrm{Aa}$ & $23,5 \mathrm{Aa}$ & $23,5 \mathrm{Aa}$ & $190,2 \mathrm{Aa}$ & $186,0 \mathrm{Aa}$ \\
\hline
\end{tabular}

Médias seguidas por diferentes letras, maiúscula na linha e minúscula na coluna, dentro de cada dimensão, diferem estatisticamente ao teste de $t$ de Student ( $p<$ 0,05). Grupo A: psicoterapia de grupo. Grupo B: controle.

$\mathrm{Na}$ dimensão Interpessoal, quando o grupo A foi comparado nos momentos 1 e 2, apresentou aumento significativo de sua média (tabela 1). Igue et. al. (2008) defendem que dados como esses são importantes de ser considerados, uma vez que as relações interpessoais permeiam todo o processo acadêmico e contribuem para o seu sucesso. A dimensão Interpessoal, entre as cinco dimensões avaliadas, foi a que apresentou maior diferença significativa no grupo A entre os momentos 1 e 2 , o que pode ser explicado pelo fato de esta ser a dimensão mais evidenciada na psicoterapia de grupo.

As dimensões pessoal e interpessoal podem ser desenvolvidas a partir da participação em psicoterapia de grupo. Segundo Rogers (1972), o ser humano desenvolve-se e existe através da interação com os outros. Erthal (2013) também pontua que o indivíduo se faz na medida em que ele é feito pela situação e pelos acontecimentos. Ao mesmo tempo em que ele se define, é definido por outro e nessa mescla de passividade e atividade, precisa reinventar-se sempre. Sendo assim, os clientes descobrem na terapia de grupo a possibilidade de lançar mão do relacionamento para sua mudança e desenvolvimento pessoal.

As médias referentes à dimensão Carreira, não diferiram significativamente em nenhuma das comparações realizadas, apesar do grupo A ter elevado sua média no momento 2 e o grupo B ter reduzido.

Em relação à dimensão Estudo, no momento 1, a média do grupo $B$ foi maior que a do grupo $A$, mas não apresentaram diferença significativa, o que é possível observar na tabela 1 . No momento 2 , ocorreu resultado semelhante, havendo uma redução na média do grupo B. Indicando assim que o grupo que não participou da 
intervenção teve uma tendência a reduzir sua adaptação em relação à dimensão estudo. Ferreira (2009) também verificou esta tendência relatando aumento de cerca de $28 \%$ no rendimento acadêmico de alunos que participaram de psicoterapia declarando seu efeito positivo nesta dimensão.

As médias referentes à dimensão Institucional não diferiram significativamente em nenhuma das comparações realizadas, apesar de, ao comparar o grupo A nos momentos 1 e 2, ter-se observado uma diferença positiva e não significativa, enquanto o grupo $B$ não apresentou nenhuma diferença.

Em relação ao processo de adaptação acadêmica, na sua totalidade, foi possível observar (tabela 1 ) que as médias dos grupos $A$ e $B$, no início do estudo, não eram significativamente diferentes, mesmo sendo a média do grupo B maior que a do grupo A. Esse resultado pode ser justificado pela forma de seleção da amostra, que foi realizada mediante um sorteio entre os participantes que se voluntariaram para frequentar a psicoterapia. No momento 2, as médias dos grupos $A$ e $B$, não apresentaram diferenças significativas. Observou-se ainda que a percepção do grupo B acerca de sua adaptação acadêmica não diferiu significativamente, apesar de ter apresentado redução. Entretanto, no grupo A, encontramos diferença significativa de elevação da percepção sobre a adaptação acadêmica, após a participação em psicoterapia de grupo (tabela 1), evidenciando que a mesma favoreceu a adaptação acadêmica.

No estudo de Casanova et. al. (2010), que buscou avaliar a percepção dos alunos acerca da adaptação em dois momentos diferentes, sem a influência de nenhum tipo de intervenção, constatou-se que houve diferença significativa e que a taxa de adaptação reduziu com a passagem do tempo para a maioria dos participantes, o que nos permite valorizar a importância da psicoterapia no processo de adaptação acadêmica.

Apesar dos valores de algumas dimensões não apresentarem médias significativas, observou-se, neste estudo, que a tendência de adaptação do grupo $A$ se elevou em todas as dimensões. Em contrapartida, a tendência de adaptação do grupo B em algumas dimensões diminuiu e em outras se manteve neutra, como pode ser verificado na Figura 1. 

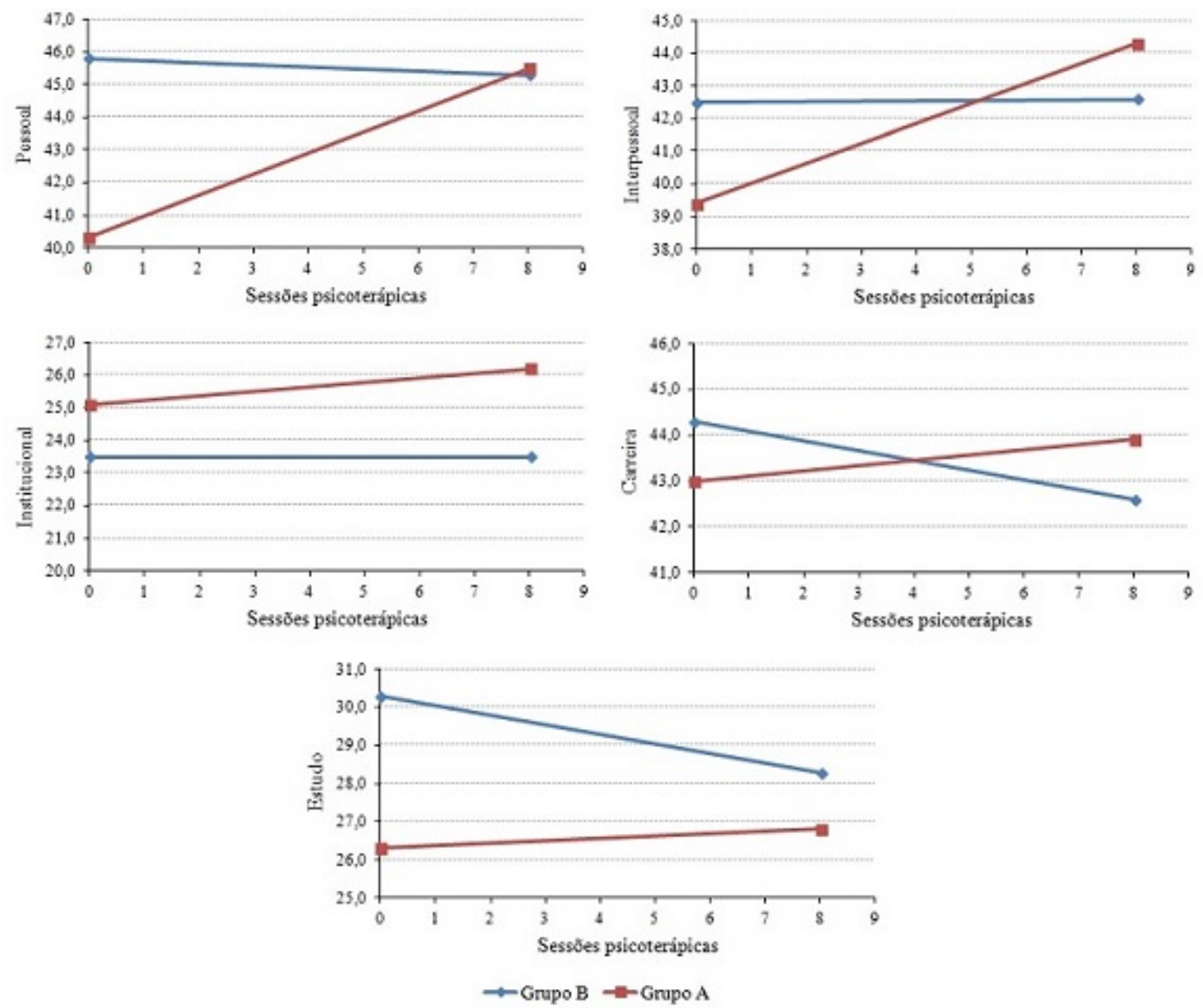

Figura 1: Desenvolvimento de adaptação acadêmica em função da participação em psicoterapia de grupo em dois momentos.

Na dimensão Pessoal, após a psicoterapia de grupo foi observada uma variação positiva na média do grupo $A(5,2$ pontos), enquanto que no grupo $B$ houve uma diminuição de $-0,5$ pontos no índice desta dimensão. Na dimensão Interpessoal, após a psicoterapia de grupo foi observada uma variação positiva no índice do grupo $A$, gerando o aumento de 4,9 pontos, enquanto no grupo $B$, o aumento no índice desta dimensão foi de 0,1 pontos (Figura 1 ). Essas duas dimensões foram as mais afetadas pela psicoterapia de grupo, pois foram as que mais variaram positivamente. De acordo com Dias (2006), Ferreira (2009) e Linhares e Meneses (2012), a resolução de dificuldades pessoais e interpessoais contribui não só para o amadurecimento psicossocial, mas também para o rendimento acadêmico, facilitando o processo de adaptação a universidade.

De modo geral, nas dimensões Carreira, Estudo e Institucional, após a psicoterapia de grupo, foi observada uma variação positiva nas médias do grupo $A(0,9 ; 0,5 ; 1,1$ pontos, respectivamente), enquanto no grupo $B$ houve diminuição $(-1,7 ;-2,0$ pontos) e na dimensão institucional se manteve igual (Figura 1). O sucesso acadêmico do aluno se inicia com o bom êxito no processo de 
adaptação e com a psicoterapia, conforme aponta Ferreira (2009), indicando que esta pode oferecer condições para que o estudante desenvolva seu potencial pessoal e acadêmico, trabalhando direta ou indiretamente questões relacionadas com as vivências acadêmicas, 0 que engloba questões das dimensões institucional, carreira e estudo.

Nos trabalhos que utilizaram o QVAr, não foi encontrada uma classificação para identificar uma gradação na adaptação acadêmica dos respondentes. Assim, neste estudo, foi proposta uma escala geral de mensuração do nível de adaptação acadêmica com base no número de itens, no valor e na quantidade das alternativas oferecidas pelo instrumento. Para tal, considerou-se o limite inferior de cinquenta e cinco pontos, baseado no somatório do menor valor possível de resposta para cada um dos itens; e o limite superior de 275 pontos, com base na soma do maior valor para cada item. Foram estabelecidos cinco níveis de adaptação: Nada adaptado (55-99 pontos), Pouco adaptado (100-143 pontos), Médio adaptado (144187 pontos), Bastante adaptado (188-231 pontos) e Totalmente adaptado (232-275 pontos).

Ao posicionar-se às médias dos grupos $A$ e $B$ nos dois momentos em que foram avaliados na escala elaborada, observou-se que o grupo $A$ no momento 1 estava médio adaptado e no momento 2 progrediu tornando-se bastante adaptado. Enquanto no grupo B, o resultado foi inverso, de bastante adaptado, no momento 1 , regrediu tornando-se médio adaptado no momento 2 . Essa observação aponta um movimento crescente do grupo A e decrescente do grupo B em direção à adaptação acadêmica (Figura 2).

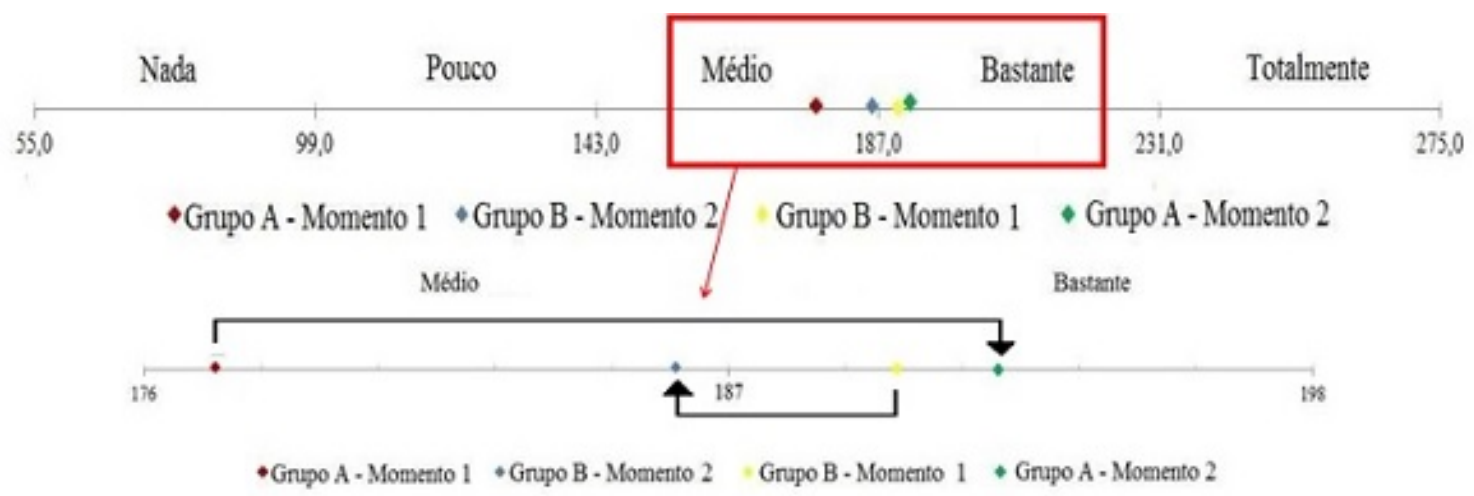

Figura 2: Escala de adaptação acadêmica.

Observa-se na figura 2, que o deslocamento do grupo A na escala foi maior que o deslocamento do grupo B. Este resultado indica que a psicoterapia de grupo pode ter impulsionado o processo de adaptação, uma vez que o intervalo de tempo foi igual para os grupos.

Em relação ao tempo em psicoterapia, avalia-se que talvez oito sessões psicoterápicas tenham sido insuficientes para promover a 
adaptação total dos participantes. Investigações sobre intervenção psicoterapêutica tem demonstrado que, após sete sessões, pelo menos 50 \% dos clientes apresentam melhora, após catorze sessões, $75 \%$, e para que haja mudanças de comportamentos em casos mais graves, vinte e uma sessões (Lambert, Hansen \& Finch, 2001; Ferreira, 2009). Com isso, podemos inferir que se o número de sessões realizadas nesse estudo tivesse sido maior, o grupo A poderia ter elevado suas médias, se mantivessem as tendências apresentadas. A elevação de cada uma das dimensões poderia gerar um aumento no nível geral de adaptação do grupo, proporcionando assim um deslocamento ainda mais positivo na escala proposta.

\section{Conclusão}

Com base nos resultados obtidos neste estudo, verificou-se que o grupo que participou da psicoterapia, ao final do estudo, elevou seu nível de adaptação acadêmica, passando de médio adaptado para bastante adaptado, enquanto o grupo controle, além de não ter apresentado aumento de média significativo em nenhuma das dimensões investigadas, ainda regrediu seu nível de adaptação, indo de bastante adaptado para médio adaptado. É possível concluir que participação engajada em um grupo psicoterápico pode auxiliar o indivíduo a enfrentar e elaborar seu processo de adaptação acadêmica no 1 을 ano de graduação.

Em relação à adaptação acadêmica integral, acredita-se que a psicoterapia de grupo pode possibilitar a aceleração da mudança de percepção dos universitários. Os dois grupos que participaram desse estudo tiveram deslocamentos diferentes no mesmo intervalo de tempo. O grupo que participou da psicoterapia teve um deslocamento maior, com movimento crescente. O grupo controle teve um deslocamento menor, como movimento decrescente. Sendo possível concluir que o deslocamento do grupo que recebeu a intervenção psicoterápica foi acelerado positivamente.

Em relação às dimensões específicas da adaptação, a psicoterapia de grupo proporcionou o desenvolvimento de capacidades referentes a essas dimensões em particular. As dimensões Pessoal e Interpessoal foram as que mais evoluíram a partir da psicoterapia, viabilizando o desenvolvimento de características como a empatia, tolerância às diferenças, maior compreensão dos sentimentos, otimismo, organização do pensamento, apoio entre pares, fortalecimento de vínculos, confiança, certeza sobre o curso escolhido e interesse pela universidade.

Apontamos como fator limitante desse estudo, o número reduzido da amostra, que produziu dificuldades em relação ao tratamento estatístico. Outro agente limitador foi a escassez de estudos similares 
ou que relacionassem o processo de adaptação com intervenção grupal, que impediu a comparação direta com resultados de outros estudos.

Como sugestões para próximos estudos, propõe-se o aumento da quantidade de sessões psicoterápicas, a fim de se evidenciar melhor os resultados.

Salienta-se a importância de que mais estudos sejam realizados envolvendo a psicoterapia de grupo e o processo de adaptação acadêmica, para que projetos de intervenção para estudantes de graduação possam ser elaborados e financiados, uma vez que verificamos que a psicoterapia de grupo é apropriada ao contexto acadêmico e que a baixa adaptação à universidade pode gerar consequências indesejáveis, tanto para os estudantes, como para as instituições universitárias.

\section{Referências}

Almeida, L. S., Ferreira, J. A. G., \& Soares, A. P. C. (1999). Questionário de Vivências Académicas: Construção e validação de uma versão reduzida (QVA-r). Revista Portuguesa de Pedagogia, 33(3), 181-207.

Almeida, L. S., Soares, A. P. C., \& Ferreira, J. A. (2002). Questionário de Vivências Acadêmicas (QVA-r): Avaliação do ajustamento dos estudantes universitários. Avaliação Psicológica, 2(1), 8193.

Bardagi, M. P. (2007). Evasão e comportamento vocacional de universitários: estudos sobre o desenvolvimento da carreira na graduação (Tese de doutorado). Universidade do Rio Grande do Sul, Porto Alegre.

Bardagi, M. P., \& Hutz, C. S. (2005). Evasão universitária e serviços de apoio ao estudante: uma breve revisão da literatura brasileira. Psicologia Revista, 14(2), 279-301.

Bardagi, M. P. \& Hutz, C. S. (2012). Mercado de trabalho, desempenho acadêmico e o impacto sobre a satisfação universitária. Revista de Ciências Humanas, 46(1), 183-198.

Bechelli, L. P. C., Santos, M. A. (2002). Psicoterapia de grupo e considerações sobre o paciente como agente da própria mudança. Revisat Latino-americana de Enfermagem, 10(3), 91383.

Bock, S. D. (2001). Orientação Profissional: avaliação de uma proposta de trabalho na abordagem sócio histórica (Dissertação de Mestrado). Universidade Estadual de Campinas, Campinas, São Paulo. 
Carmo, M. C., \& Polydoro, S. A. J. (2010). Integração ao ensino superior em um curso de Pedagogia. Psicologia Escolar e Educacional, 14(1), 211-220.

Casanova, D. G., \& Polydoro, S. (2010). Integração ao ensino superior: Relações ao longo do primeiro ano de graduação. Psicologia: Ensino \& Formação, 1(2), 85-96.

Cunha, S. M., \& Carrilho, D. M. (2005). O processo de adaptação ao ensino superior e o rendimento acadêmico. Psicologia Escolar e Educacional, 9(2), 215-224.

Dias, G. F. (2006). Aconselhamento psicológico a jovens do Ensino Superior: Uma abordagem psicodinâmica e desenvolvimentista. Análise Psicológica, 24(1), 39-50.

Diniz, A. M., \& Almeida, L. S. (2006). Adaptação à universidade em estudantes de primeiro ano: estudo diacrónico da interação entre o relacionamento com pares, o bem-estar pessoal e o equilíbrio emocional. Análise Psicológica, 24(1), 29-38.

Enéas, M. L. E., Faleiros, J. C., \& Sá, A. C. A. (2000). Uso de psicoterapias breves em clínica-escola: caracterização dos processos com adultos. Psicologia: Teoria e Prática, 2(2), 9-30.

Erthal, T. C. S. (2013). Trilogia da existência: teoria e prática da psicoterapia vivencial. Curitiba: Appris.

Feitosa, M. G. G. (2001). Integração de estudantes ingressantes à vida universitária: fatores que facilitam e que dificultam sua integração (Tese de Doutorado). Universidade Federal de São Carlos, Faculdade de Educação, São Carlos, São Paulo.

Fernandes, E., \& Almeida, L. (2005). Expectativas e vivências acadêmicas em estudantes do $1^{\circ}$ ano: diferenças de gênero, situação de estudante e curso. Psico-USF, 6(1), 1-10.

Ferreira, C. A. M. (2009). Intervenção psicológica no ensino superior: efeito da psicoterapia no rendimento acadêmico (Dissertação de Mestrado). Universidade de Lisboa, Lisboa.

Ferreira, J. A., Almeida, L. S. \& Soares, A. P. (2001). Adaptação acadêmica em estudantes do 1 o ano: diferenças de gênero, situação de estudantes e cursos. Revista Psico-USF, 6(1), 1-10.

Granado, J. I. F., Santos, A. A. A., Almeida, L. S., Soares, A. P., \& Guisande, M. A. (2005). Integração acadêmica de estudantes universitários: Contributos para a adaptação e validação do QVA-r no Brasil. Psicologia e Educação, 4(1), 33-43.

Howard, K. I., Kopta, S. M., Krause, M. S., \& Orlinsky, D. E. (1986). The dose-effect relationship in psychotherapy. American Psychologist, 41, 159-164.

Igue, E. A., Bariani, I. C. D., \& Milanesi, P. V. B. (2008). Vivência acadêmica e expectativas de universitários ingressantes e concluintes. Psico-USF, 13(2), 155-164.

Lambert, M. J., Hansen, N. B., \& Finch, A. E. (2001). Patient-focused research: using patient outcome date to enhance treatment 
effects. Journal of Consulting and Clinical Psychology, 69(1), 157-172.

Linhares, V., \& Meneses, R. F. (2012). Desenvolvimento de competências psicossociais: uma experiência de intervenção em grupo. In II Congresso nacional RESAPES AP, Porto. Anais Apoio psicológico no ensino superior: um olhar sobre o futuro.

Marinho-Araujo, C. M., Fleith, D. S., Almeida, L. S., Bisinoto, C., \& Rabelo, M. L. (2015). Adaptação da Escala Expectativas Acadêmicas de Estudantes Ingressantes na Educação Superior. Avaliação Psicológica, 14(1), 133-141.

Meier, M. J., \& Kudlowiez, S. (2003). Grupo focal: uma experiência singular. Texto \& Contexto Enfermagem, 12(3), 394-399.

Militão, A., \& Militão, R. Jogos, dinâmicas e vivências grupais. Rio de J aneiro: Qualitymark Editora, 2000.

Ministério da Educação. (2014). Senso da Educação Superior: 2013. Retirado

de http://download.inep.gov.br/educacao_superior/censo_superior /apresentacao/2014/coletiva_censo_superior_2013.pdf

Moraes, M. A. (2003). Psicologia como reflexão sobre as práticas humanas: da adaptação à errância. Estudos de psicologia, 8(3), 535-539.

Oliveira, M. L. C., Dantas, C. R., Azevedo, R. C. S., \& Banzato, C. E. M. (2008). Demographics and complaints of university students who sought help at a campus mental health service between 1987 and 2004. São Paulo Medical J ournal, 126(1), 58-62.

Oliveira, R., \& Morais, A. (2015). Vivências acadêmicas e adaptação de estudantes de uma universidade pública federal do estado do Paraná. Revista De Educação Pública, 24(57), 547-568.

Palma, A. M. P. V., Palma, S. P. V., \& Brancaleoni, A. P. L. (2005). Prevenção à evasão no ensino superior: necessária implementação de orientação profissional a graduandos em desligamento. In M. C. P. Lassance et al. (Eds.), Intervenção e compromisso social: Orientação profissional - teoria e técnica (Vol. 2, pp. 303-320). São Paulo: Vetor.

Pascarella, E. T., \& Terenzini, E. T. (2005). How college affects students: A third decade of research (Vol. 2). San Francisco: Jossey-Bass.

Pereira, A. M. S., Motta, E. D., Vaz, A. L., Pinto, C., Bernardino, O., Melo, A. C., Ferreira, J., Rodrigues, M. J., Medeiros, A., \& Lopes, P. N. (2006). Sucesso e desenvolvimento psicológico no Ensino Superior: Estratégias de intervenção. Análise Psicológica, 24(1), 51-59.

Rogers, C. (1972). Tornar-se pessoa. Lisboa: Moraes.

Santos, A. A. A., Noronha, A. P. P., Amaro, C. B., \& Villar, J. (2005). Questionário de Vivência Acadêmica: Estudo da consistência interna do instrumento no contexto brasileiro. In M. C. R. A. 
Joly, A. A. A. Santos \& F. F. Sisto (Eds.), Questões do cotidiano universitário (pp. 159-177). São Paulo: Casa do Psicólogo.

Sarriera, J. C., Paradiso, A. C., Schütz, F. F. \& Howes, G. P. (2012). Estudo comparativo da integração ao contexto universitário entre estudantes de diferentes instituições. Revista Brasileira de Orientação Profissional, 13(2), 163-172.

Sartre, J. P. (2005). A Náusea (12 ${ }^{a}$ ed.). Rio de Janeiro: Nova Fronteira.

Serrão, M., \& Baleeiro, M. C. (1999). Aprendendo a Ser e a Conviver. Salvador, BA: FTD.

Soares, A. P., Almeida, L. S., Diniz, A. M., \& Guisande, M. A. (2006). Modelo Multidimensional de Ajustamento de jovens ao contexto Universitário (MMAU): Estudo com estudantes de ciências e tecnologias versus ciências sociais e humanas. Análise Psicológica, 24(1), 15-27.

Soares, A. B., \& Prette, Z. A. P. D. (2015). Habilidades sociais e adaptação à universidade: Convergências e divergências dos construtos. Análise Psicológica, 33(2), 139-151.

Sousa, D. C. M. D. (2006). Investigação em psicoterapia: Contexto, questões e controvérsias: Possíveis contributos da perspectiva fenomenológico existencial. Análise Psicológica, 24(4), 373382.

Teixeira, M. A. P., Dias, A. C. G., Wothich, S. H., \& Oliveira, A. M. (2008). Adaptação à universidade em jovens calouros. Psicologia Escolar e Educacional, 12(1), 185-202.

Villar, J. D. (2001). Adaptação de questionário de vivência universitária com estudantes de Arquitetura e de Engenharia (Dissertação de Mestrado). Universidade São Francisco, Itatiba, São Paulo.

Yalom, I. D., \& Leszcz, M. (2007). Psicoterapia de grupo: teoria e prática. Porto Alegre: Artmed.

Zimerman, D. E. (2000). Fundamentos básicos das grupoterapias. Porto Alegre: Artes Médicas.

\author{
Endereço para correspondência \\ Daiane Bocard do Couto \\ Universidade Federal Rural do Rio de Janeiro - UFRRJ \\ Instituto de Educação - IE \\ Departamento de Psicologia - DEPSI (Sala 15) \\ Rodovia BR 465, Km 07, s/n, Zona Rural, CEP 23890-000, Seropédica - RJ, Brasil \\ Endereço eletrônico: daianebocard@gmail.com

\section{Carla Cristine Vicente} \\ Universidade Federal Rural do Rio de J aneiro - UFRRJ \\ Instituto de Educação - IE \\ Departamento de Psicologia - DEPSI (Sala 15) \\ Rodovia BR 465, Km 07, s/n, Zona Rural, CEP 23890-000, Seropédica - RJ, Brasil \\ Endereço eletrônico: carlavicent@gmail.com
}


Recebido em: 16/12/2015

Reformulado em: 07/10/2018

Aceito em: 08/10/2018

\section{Notas}

* Mestre em Psicologia pela Universidade Federal Rural do Rio de Janeiro (UFFRJ) e Psicóloga formada pela UFRRJ .

** Doutora em Psicologia Social pela Universidade do Estado do Rio de Janeiro (UERJ). Docente da Universidade Federal Rural do Rio de Janeiro (UFRRJ) na área da Psicologia do Desenvolvimento Humano e Dinâmica de Grupos. Atualmente supervisiona estágio profissional em psicologia de grupos e projetos de extensão de grupos na perspectiva Fenomenológico Existencial.

Este artigo de revista Estudos e Pesquisas em Psicologia é licenciado sob uma Licença Creative Commons Atribuição-Não Comercial 3.0 Não Adaptada. 